Int. J. Dev. Biol. 63: 615-621 (2019)

https://doi.org/10.1387/ijdb.190049rp

\title{
Expression of primary cilia-related genes in developing mouse gonads
}

\author{
RAFAL P. PIPREK *,1, DAGMARA PODKOWA', MALGORZATA KLOC ${ }^{2,3,4}$ and JACEK Z. KUBIAK5,6 \\ ${ }^{1}$ Department of Comparative Anatomy, Institute of Zoology and Biomedical Research, Jagiellonian University, Krakow, Poland, \\ ${ }^{2}$ The Houston Methodist Research Institute, Houston, TX, USA, ${ }^{3}$ Department of Surgery, The Houston Methodist Hospital, \\ Houston TX, USA, ${ }^{4}$ University of Texas, MD Anderson Cancer Center, Houston TX, USA, ${ }^{5}$ Univ Rennes, CNRS, Institute of \\ Genetics and Development of Rennes, UMR 6290, Cell Cycle Group, Faculty of Medicine, Rennes, France and ${ }^{6}$ Laboratory of \\ Regenerative Medicine and Cell Biology, Military Institute of Hygiene and Epidemiology (WIHE), Warsaw, Poland
}

\begin{abstract}
Mechanisms governing differentiation of the bipotential gonad into the testes or ovaries are complex and still vague. The primary cilium is an organelle involved in cell signaling, which controls the development of many organs, but the role of primary cilium in the sex determination and sexual differentiation of gonads is completely unknown. Here we studied the expression of genes involved in primary cilium formation and functioning in fetal mouse gonads, before, during and after sexual differentiation. We studied the expression of 175 primary cilia-related genes using microarray technique. 144 of these genes were ubiquitously expressed in all studied cell types with no significant differences in expression level. Such a high level of expression of primary cilia-related genes in developing mouse gonads suggests that the primary cilia and/or primary cilia-related genes are important for the development of both somatic and germline component of the gonads. Only 31 genes showed a difference in expression between different cell types, which suggests that they have different functions in the somatic and germ cells. These results justify further studies on the role of primary cilia and the primary cilia-related genes in gonad development.
\end{abstract}

KEY WORDS: gonad development, sex determination, ovary, testis, primary cilia

The testes and the ovaries develop from the bipotential gonads in the process of sexual differentiation. In the mouse, the gonadal primordia (genital ridges) appear just before $10.5^{\text {th }}$ day of embryonic life (E10.5) (Hu et al., 2013; Piprek et al., 2016). Between stage E10.5 and E12.5, the sexually undifferentiated gonads start expressing the sex-determining genes (reviewed in Piprek et al., 2016). The fate of the gonad and its ultimate differentiation into the testis or ovary depends on male or female sex-determining signaling pathways (reviewed in Piprek, 2009a, 2009b). At stage E13.5, the mouse gonads are already sexually differentiated, and their sex can be easily recognized under the microscope (Nel-Themaat et al., 2009). Developing gonads are composed of three basic cell types: i) supporting cells (Sertoli and follicular cells), ii) interstitial/stromal cells, and iii) germ cells (Piprek et al., 2017, 2018). Although a number of genes and signaling pathways (such as PDGF - platelet-derived growth factor pathway, FGF - fibroblast growth factor pathway, WNT - wingless-type MMTV integration site family pathway, and
Hedgehog pathway) involved in sex determination and sexual differentiation of mouse gonad have been identified (reviewed in Piprek, 2009a,b, 2010), the mechanisms directing bipotential gonad differentiation into the testes or ovaries are very complex, and thus still require further studies.

Studies of the last decade identified the primary cilium as a key coordinator of signaling during embryogenesis and organogenesis (Satir et al., 2010). The primary cilium is an immotile organelle present on the surface of a large variety of eukaryotic cells. The primary cilium contains peripheral doublets of microtubules and lacks central microtubules (9+0 axoneme pattern). The primary cilium disappears during cell division. Recently, it has been shown that the primary cilium possesses various receptors and acts as "a cell's antenna", which enables the cell to respond to various signaling molecules (reviewed in Wainwright et al., 2014). Recently, the genes important for primary cilium formation and function,

Abbreviations used in this paper: FGF, fibroblast growth factor.

\footnotetext{
*Address correspondence to: Rafal P. Piprek. Department of Comparative Anatomy, Institute of Zoology and Biomedical Research, Jagiellonian University, Gronostajowa 9, 30-387 Krakow, Poland. Tel: +48126645059. E-mail: rafal.piprek@uj.edu.pl - (iD) https://orcid.org/0000-0002-0018-2444
} 
have been identified and characterized (Ishikawa et al., 2012). The fact that primary cilium participates in receiving signals from PDGF, FGF, WNT and Hedgehog pathways suggests that this organelle may be important for gonad development. The expression of many primary cilia-related genes in developing mouse gonads suggests that primary cilia or primary cilia-related mRNAs and proteins are present in three studied cell types and may play a role in the differentiation of these cells and in sexual differentiation of gonads. Very little is known about the presence of primary cilia in the gonads. Wainwright and colleagues (2014) showed that the primary cilia are present in the somatic and germ cells of fetal mouse gonads between stage E10.5 and E13.5 (Wainwright et al., 2014). However, from stage E13.5 onward only interstitial (Leydig and peritubular myoid) cells retain primary cilia, and no primary cilia are present in the Sertoli or germ cells (Wainwright et al., 2014). Also in the adult human testis, the primary cilia are only present in the Leydig and peritubular myoid cells (Nygaard et al., 2015). In contrast, in pig developing testes, the primary cilia were detected in Sertoli cells and interstitial cells, but not in the germ cells (Ou et al., 2014). However, there are no studies on the role of the primary cilium in sex determination and sexual differentiation of gonads. Here we studied the expression of primary cilium-related genes in supporting cells, interstitial/stromal cells and germ cells isolated from developing mouse gonads at three developmental stages: E11.0 (the sex determination period), E12.2 (the onset of sexual differentiation), E13.8 (sexually differentiated gonads), using microarray technique supported by qPCR.

\section{Results and Discussion}

In 2012, Ishikawa and collaborators identified 195 primary ciliarelated genes expressed in mouse kidney cells (Ishikawa et al., 2012). Here, we detected the expression of 175 of these genes in developing mouse gonads (Table 1,2,3,4). 144 of these genes were ubiquitously expressed in all studied cell types at E11.0, E12.2, and E13.8 with no significant differences in expression level (Table 1), and only 31 genes showed the difference in the expression between cell types. Twenty-five of these genes had a different level of expression between the somatic cells and the germ cells. Among these genes, 12 genes had a higher expression (Table 2), and 13 genes had lower expression in the germ cells (Table 3 ) comparing to the somatic cells. Only 6 genes showed differences in the expression level between supporting and interstitial/stromal cells (Table 4). qPCR analysis of 8 genes expression confirmed results of microarray analysis (Fig. 1).

Among the primary cilia-related genes ubiquitously expressed in developing mouse gonads with no significant differences between cell types (fold change < 1.5) were (144 genes) for example: ADP-ribosylation factors (Arf), ADP ribosylation factor like GTPase 13B $(A r / 13 b)$, calumenin (Calu), chaperonin containing t-complex polypeptides (Cct), calponin 3 (Cnn3), exportin 2 (Cse1), cullin2 (Cul2), dynactin 2 (Dctn2), dynamin 1-like protein (Dnm 1), dynamin 2 (Dnm2), cytoplasmic dyneins (Dync), eukaryotic translation initiation factors (Eif), intraflagellar transport proteins (Ift), importin 7 (Ipo7), kinesin family members (Kif), nucleolin ( Ncl), nardilysin (Nrd1), prostaglandin E synthase 3 (Ptges3), Ras-related proteins (Rab), septins (Sept), and exportin 9 (Xpo7); Table 1. A function of ADP ribosylation factor like GTPase $13 \mathrm{~B}(A r / 13 b)$ is restricted to the primary cilia. This GTPase is localized in the cilia and plays a role in cilia formation and their maintenance (Higginbotham et al., 2012). Other genes important for the primary cilia are intraflagellar transport proteins Ift20, Ift88, and Ift172. They are responsible for cilium biogenesis. The ubiquitous expression of these genes in all cell types of developing mouse gonad suggests that, at certain point in development, all these cells contain primary cilia. Indeed, the majority of the above genes are known to play other, broader than cilia-related functions in a cell. This again indicates that the functions of these genes in the developing gonad may be broader and not limited to the primary cilia.

Among primary cilia-related genes expressed at significantly higher level (fold change $\geq 1.5$ ) in the germ cells than in somatic cells were (12 genes): arsenical pump-driving ATPase (Asna1), protein diaphanous homolog 1 (Diap1), insulin-degrading enzyme (Ide), protein phosphatase 2 regulatory subunit $\mathrm{A}$ beta (Ppp2r1b), peroxiredoxin 4 (Prdx4), 60S ribosomal protein L12 (Rp/12), $60 \mathrm{~S}$ acidic ribosomal protein P2 (Rp/p2), ribosomal protein S12 (Rps12), testis-specific gene A14 (Tsga14), tubulin alpha-4A chain (Tuba4a), and exportin 5 (Xpo5); Table 2. The Solute carrier family 2 member 1 (S/c2a1) was the only gene showing a significant difference in the expression level between $X X$ and $X Y$ germ cells, with the higher expression in the $X Y$ germ cells (Table 2 ), which suggests that this gene product may be involved in differentiation of spermatogonia.

Among primary cilia-related genes expressed at significantly lower level (fold change $\geq 1.5$ ) in the germ cells than in somatic cells were (13 genes): ADP-ribosylation factor-like protein 3 (Arl3), Calcium/calmodulin-dependent protein kinase type II delta (Camk2d), calpain-2 catalytic subunit (Capn2), Erbb2 interacting protein (Erbb2ip), GNAS (guanine nucleotide-binding protein, alpha stimulating) complex locus (Gnas), isocitrate dehydrogenase 1 (Idh1), peroxiredoxin $3(\operatorname{Prd} x 3)$, protein tyrosine phosphatase non-receptor type 13 (Ptpn13), protein transport protein Sec23A (Sec23a), septin 9 (Spet9), triosephosphate isomerase 1 (Tpi1), tetratricopeptide repeat protein $30 \mathrm{~B}$ (Ttc30b), and tubulin beta-2A chain (Tubb2a); Table 3.

Among primary cilia-related genes showing significant differences in the expression level (fold change $\geq 1.5$ ) between different type or sex of somatic cells were (6 genes): Ran GTPase-activating protein 1 (Rangap1), protein transport protein Sec23B (Sec23b), syntrophin basic 2 (Sntb2), transmembrane protein 2 (Tmem2), and tubulin beta-3 chain (Tubb3). These genes showed higher expression in $X Y$ than in $X X$ supporting cells (Table 4). This suggests that these genes may be involved in sex determination and/or sexual differentiation of supporting cells. Another tubulin gene, tubulin beta-6 chain (Tubb6), had a higher level of expression in the interstitial/stromal cells than in the supporting cells (Table 4), which suggests its importance for differentiation of the interstitial/stromal cells.

As mentioned above, the primary cilia were previously detected in the somatic cells of developing mouse, pig, and human gonads but they were absent in the germ cells (Nygaard et al., 2015; Ou et al., 2014; Wainwright et al., 2014). Presented here the global analysis of expression showed the expression of primary cilia-related genes in both somatic and germ cells during the sex determination and sexual differentiation period of the gonad. Further studies are necessary to establish if the germ cells in fetal mouse gonad possess primary cilia at a certain stage(s) and if there are any differences in the function of primary cilia or 


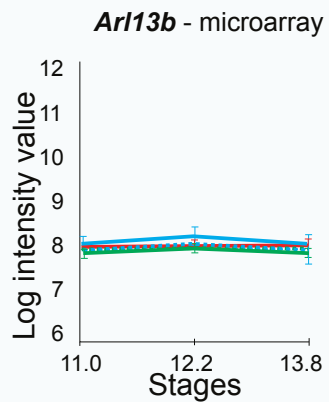

Ift20 - microarray

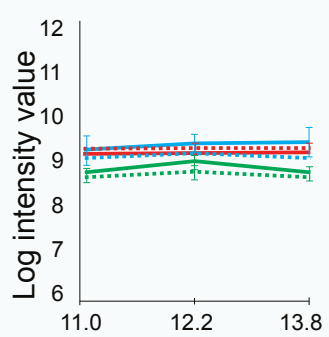

Tuba4a - microarray

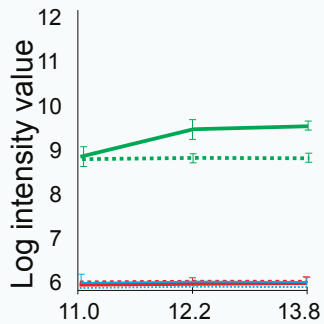

Tubb3 - microarray

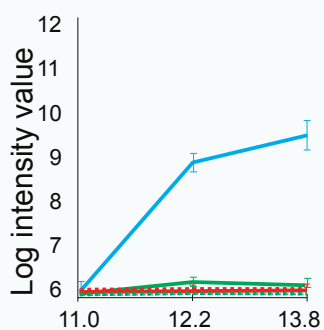

Arl13b - qPCR

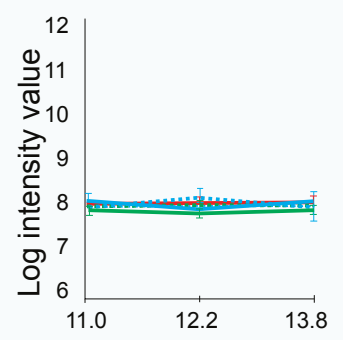

Ift20 - qPCR

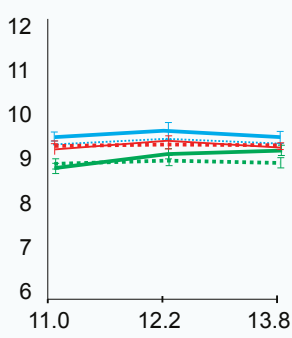

Tuba4a - qPCR

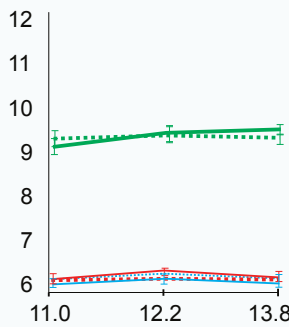

Tubb3 - qPCR

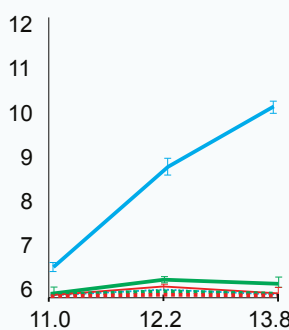

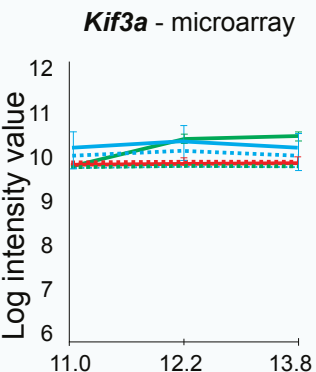

Ift88 - microarray

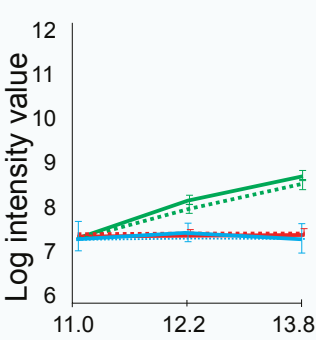

Tubb2a - microarray

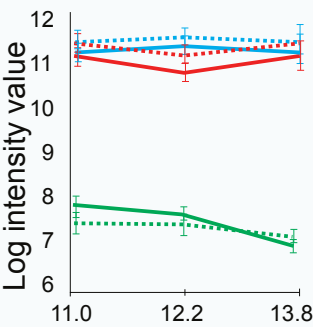

Actb - microarray

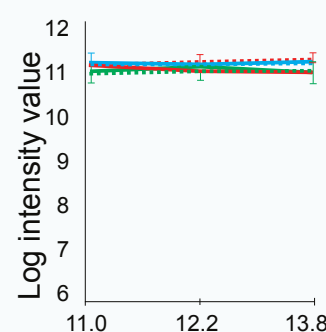

Kif3a - qPCR

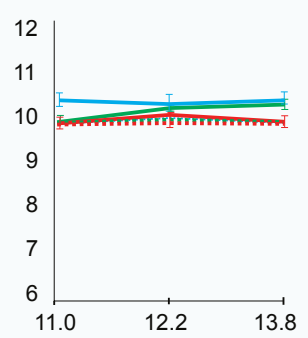

Ift88 - qPCR

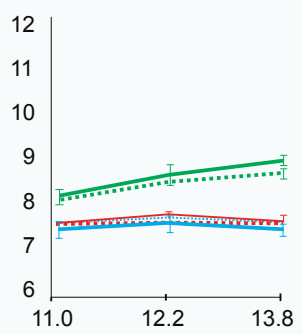

Tubb2a - qPCR

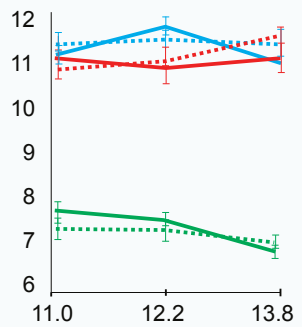

Actb - qPCR

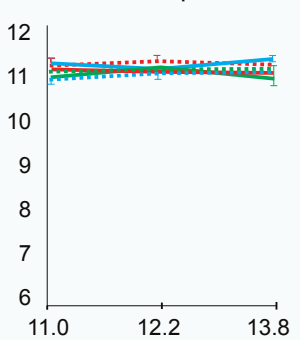

Fig. 1. Graphs of microarray validation. Comparison of the log-normalized intensity values (6-12) of the expression of primary-cilia related genes Arl13b, Kif3a, Ift20 expressed ubiquitously at high level in three studied cell lines, Ift88, Tuba4a showing higher expression in the germ cells, Tubb2a showing lower expression in the germ cells, Tubb3 showing higher expression in $X Y$ supporting cells, and Actb as a positive control gene obtained via microarray supported by qPCR. 
primary cilia-related genes in different cell lines in differentiating gonads. Differences in the expression of primary cilia-related genes between somatic and germ cells suggest that, indeed, there is a difference in the function of primary cilia or primary cilia-related genes between somatic and germ cells. Wainwright and colleagues (2014) showed that mice with a mutation in Ift144 (intraflagellar transport gene 144) gene had abnormally large gonads and more testis cords than control gonad (Wainwright et al., 2014). This

\section{TABLE 1}

PRIMARY CILIA-RELATED GENES EXPRESSED UBIQUITOUSLY IN THE DEVELOPING MOUSE GONADS WITH NO SIGNIFICANT DIFFERENCES BETWEEN CELL TYPES

\begin{tabular}{|c|c|c|c|c|c|c|c|}
\hline \multirow[b]{2}{*}{ Gene symbol } & \multirow[b]{2}{*}{ Gene name } & \multicolumn{2}{|c|}{ Supporting cells } & \multicolumn{2}{|c|}{ Interstitial/stromal cells } & \multicolumn{2}{|c|}{ Germ cells } \\
\hline & & $\mathbf{x X}$ & $\mathbf{X Y}$ & $\mathbf{x x}$ & $\mathbf{X Y}$ & $\mathbf{x X}$ & $\mathbf{X Y}$ \\
\hline Abce1 & ATP-binding cassette sub-family E member 1 & +++ & +++ & +++ & +++ & +++ & +++ \\
\hline Acaca & Acetyl-Coenzyme A carboxylase alpha & + & + & + & + & + & + \\
\hline Adpgk & ADP-dependent glucokinase & + & + & + & + & + & + \\
\hline Aldh18a1 & Aldehyde dehydrogenase 18 family, member $\mathrm{A} 1$ & . & . & . & . & . & . \\
\hline Anp32e & Acidic leucine-rich phosphoprotein 32 member $E$ & +++ & +++ & +++ & +++ & +++ & +++ \\
\hline$A p 2 b 1$ & AP-2 complex subunit beta-1 & ++ & ++ & ++ & ++ & ++ & ++ \\
\hline Arf4 & ADP-ribosylation factor 4 & +++ & +++ & +++ & +++ & +++ & +++ \\
\hline Arf6 & ADP-ribosylation factor 6 & +++ & +++ & +++ & +++ & +++ & +++ \\
\hline Arfgef1 & ADP-ribosylation factor guanine nucleotide factor 1 & +++ & +++ & +++ & +++ & +++ & +++ \\
\hline Arhgap5 & Rho GTPase activating protein 5 & ++ & ++ & ++ & ++ & ++ & ++ \\
\hline$A r l 13 b$ & ADP-ribosylation factor-like protein $13 B$ & ++ & ++ & ++ & ++ & ++ & ++ \\
\hline Arpc3 & Actin-related protein $2 / 3$ complex subunit 3 & ++ & ++ & ++ & ++ & ++ & ++ \\
\hline Azi1 & 5-azacytidine-induced protein 1 & . & . & . & . & . & . \\
\hline B230208H17 & Putative GTP-binding protein Parf & ++ & ++ & ++ & ++ & ++ & ++ \\
\hline Btf3 & Basic transcription factor 3 & ++ & ++ & ++ & ++ & ++ & ++ \\
\hline Calu & Calumenin & +++ & +++ & +++ & +++ & +++ & +++ \\
\hline Ccdc47 & Coiled-coil domain-containing protein 47 & +++ & +++ & +++ & +++ & +++ & +++ \\
\hline Cct4 & Chaperonin containing t-complex 1 , subunit 4 & ++ & ++ & ++ & ++ & ++ & ++ \\
\hline Cct5 & Chaperonin containing t-complex 1 , subunit 5 & +++ & +++ & +++ & +++ & +++ & +++ \\
\hline Cct6a & Chaperonin containing t-complex 1 , subunit $6 a$ & +++ & +++ & +++ & +++ & +++ & +++ \\
\hline Cct8 & Chaperonin containing t-complex 1 , subunit 8 & +++ & +++ & +++ & +++ & +++ & +++ \\
\hline Chmp4b & Charged multivesicular body protein $4 \mathrm{~b}$ & ++ & ++ & ++ & ++ & ++ & ++ \\
\hline Cluap1 & Clusterin-associated protein 1 & ++ & ++ & ++ & ++ & ++ & ++ \\
\hline Cnn3 & Calponin 3 & ++ & ++ & ++ & ++ & ++ & ++ \\
\hline Cnp & 2',3'-cyclic nucleotide 3' phosphodiesterase & . & . & . & . & . & . \\
\hline $\operatorname{Cog} 4$ & Conserved oligomeric Golgi complex subunit 4 & + & + & + & + & + & + \\
\hline Copb2 & Coatomer subunit beta & +++ & +++ & +++ & +++ & +++ & +++ \\
\hline Cse1/ & Exportin 2 & +++ & +++ & +++ & +++ & +++ & +++ \\
\hline Cul2 & Cullin 2 & + & + & + & + & + & + \\
\hline D630037F22 & Broad-minded & + & + & + & + & + & + \\
\hline Daam1 & Disheveled-associated activator of morphogenesis 1 & ++ & ++ & ++ & ++ & ++ & ++ \\
\hline Dcdc2a & Doublecortin domain-containing protein $2 \mathrm{a}$ & . & . & . & . & . & . \\
\hline Dctn2 & Dynactin 2 & ++ & ++ & ++ & ++ & ++ & ++ \\
\hline Dhx30 & DEAH box polypeptide 30 & + & + & + & + & + & + \\
\hline Dnm1l & Dynamin-1-like protein & ++ & ++ & ++ & ++ & ++ & ++ \\
\hline Dnm2 & Dynamin 2 & ++ & ++ & ++ & ++ & ++ & ++ \\
\hline Drg1 & Developmentally-regulated GTP-binding protein 1 & +++ & +++ & +++ & +++ & +++ & +++ \\
\hline Drg2 & Developmentally-regulated GTP-binding protein 2 & + & + & + & + & + & + \\
\hline Dync1h1 & Cytoplasmic dynein 1 heavy chain 1 & ++ & ++ & ++ & ++ & ++ & ++ \\
\hline Dync1li1 & Cytoplasmic dynein 1 light intermediate chain 1 & ++ & ++ & ++ & ++ & ++ & ++ \\
\hline Dync2h1 & Cytoplasmic dynein 2 heavy chain 1 & + & + & + & + & + & + \\
\hline Dync2li1 & Cytoplasmic dynein 2 light intermediate chain 1 & + & + & + & + & + & + \\
\hline Edc4 & Enhancer of mRNA-decapping protein 4 & . & . & . & . & . & . \\
\hline Eef1d & Eukaryotic translation elongation factor 1 delta & ++ & ++ & ++ & ++ & ++ & ++ \\
\hline Efcab7 & EF-hand calcium-binding domain-containing protein 7 & ++ & ++ & ++ & ++ & ++ & ++ \\
\hline Eif2s2 & Eukaryotic translation initiation factor 2 subunit 2 & ++ & ++ & ++ & ++ & ++ & ++ \\
\hline Eif3b & Eif3b protein & +++ & +++ & +++ & +++ & +++ & +++ \\
\hline Eif3l & Eukaryotic translation initiation factor 3 subunit $L$ & +++ & +++ & +++ & +++ & +++ & +++ \\
\hline Eif4g1 & Eukaryotic translation initiation factor 4 , gamma 1 & ++ & ++ & ++ & ++ & ++ & ++ \\
\hline Eif4h & Eukaryotic translation initiation factor $4 \mathrm{H}$ & +++ & +++ & +++ & +++ & +++ & +++ \\
\hline Eif5b & Eukaryotic translation initiation factor $5 \mathrm{~B}$ & +++ & +++ & +++ & +++ & +++ & +++ \\
\hline Epb4.112 & Erythrocyte protein band 4.1 -like 2 & +++ & +++ & +++ & +++ & +++ & +++ \\
\hline Eps15/1 & Epidermal growth factor receptor substrate 15 -like 1 & + & + & + & + & + & + \\
\hline Etfb & Electron transfer flavoprotein subunit beta & +++ & +++ & +++ & +++ & +++ & +++ \\
\hline Fam114a2 & Family with sequence similarity 114 , member $\mathrm{A} 2$ & ++ & ++ & ++ & ++ & ++ & ++ \\
\hline Fam $49 b$ & Family with sequence similarity 49 , member B & ++ & ++ & ++ & ++ & ++ & ++ \\
\hline Flii & Flightless 1 homolog & + & + & + & + & + & + \\
\hline G3bp1 & Ras GTPase-activating protein-binding protein 1 & ++ & ++ & ++ & ++ & ++ & ++ \\
\hline Gart & Phosphoribosylglycinamide formyltransferase & ++ & ++ & ++ & ++ & ++ & ++ \\
\hline Gbf1 & Golgi-specific brefeldin A-resistance factor 1 & ++ & ++ & ++ & ++ & ++ & ++ \\
\hline Gt/3 & Gene trap locus 3 & ++ & ++ & ++ & ++ & ++ & ++ \\
\hline Hars & Putative uncharacterized protein & ++ & ++ & ++ & ++ & ++ & ++ \\
\hline Hspa1a & Heat shock protein $1 \mathrm{~A}$ & +++ & +++ & +++ & +++ & +++ & +++ \\
\hline Hspa4 & Heat shock $70 \mathrm{kDa}$ protein 4 & +++ & +++ & +++ & +++ & +++ & +++ \\
\hline Hspb1 & Heat shock protein beta-1 & . & . & . & . & . & . \\
\hline Hspb11 & Putative uncharacterized protein & ++ & ++ & ++ & ++ & ++ & ++ \\
\hline Hsph1 & Heat shock protein $105 \mathrm{kDa}$ & +++ & +++ & +++ & +++ & +++ & +++ \\
\hline Ift122 & Intraflagellar transport protein 122 homolog & . & . & . & . & . & . \\
\hline
\end{tabular}


TABLE 1 (CONTINUED)

\begin{tabular}{|c|c|c|c|c|c|c|c|}
\hline \multirow[b]{2}{*}{ Gene symbol } & \multirow[b]{2}{*}{ Gene name } & \multicolumn{2}{|c|}{ Supporting cells } & \multicolumn{2}{|c|}{ Interstitial/stromal cells } & \multicolumn{2}{|c|}{ Germ cells } \\
\hline & & $\mathbf{x X}$ & $\mathbf{X Y}$ & $\mathbf{x X}$ & $\mathbf{X Y}$ & $\mathbf{X X}$ & $\mathbf{X Y}$ \\
\hline Ift140 & intraflagellar transport 140 & + & + & + & + & + & + \\
\hline Ift172 & Intraflagellar transport protein 172 homolog & + & + & + & + & + & + \\
\hline Ift20 & Intraflagellar transport protein 20 homolog & ++ & ++ & ++ & ++ & ++ & ++ \\
\hline Ift52 & Intraflagellar transport protein 52 homolog & ++ & ++ & ++ & ++ & ++ & ++ \\
\hline Ift57 & Intraflagellar transport protein 57 homolog & ++ & ++ & ++ & ++ & ++ & ++ \\
\hline Ift74 & Intraflagellar transport protein 74 homolog & ++ & ++ & ++ & ++ & ++ & ++ \\
\hline Ift80 & Intraflagellar transport protein 80 homolog & ++ & ++ & ++ & ++ & ++ & ++ \\
\hline Ift81 & Intraflagellar transport protein 81 homolog & ++ & ++ & ++ & ++ & ++ & ++ \\
\hline Ift88 & Putative uncharacterized protein & + & + & + & + & + & + \\
\hline Inpp5e & Inositol polyphosphate-5-phosphatase E & . & . & . & . & . & . \\
\hline Invs & Inversin & + & + & + & + & + & + \\
\hline Ipo5 & Importin 5 & ++ & ++ & ++ & ++ & ++ & ++ \\
\hline Ipo7 & Importin 7 & ++ & ++ & ++ & ++ & ++ & ++ \\
\hline Kif3a & Kinesin family member $3 \mathrm{~A}$ & ++ & ++ & ++ & ++ & ++ & ++ \\
\hline Kif3b & Kinesin family member 3B & + & + & + & + & + & + \\
\hline Kifap3 & Kinesin-associated protein 3 & + & + & + & + & + & + \\
\hline Lamb1 & Laminin B1 & ++ & ++ & ++ & ++ & ++ & ++ \\
\hline Lca5 & Leber congenital amaurosis 5 & + & + & + & + & + & + \\
\hline Lrrc40 & Leucine-rich repeat-containing protein 40 & ++ & ++ & ++ & ++ & ++ & ++ \\
\hline Lrrc59 & Leucine-rich repeat-containing protein 59 & + & + & + & + & + & + \\
\hline Macf1 & Microtubule-actin cross-linking factor 1 & ++ & ++ & ++ & ++ & ++ & ++ \\
\hline Mcm4 & DNA replication licensing factor MCM4 & ++ & ++ & ++ & ++ & ++ & ++ \\
\hline Mtap1s & Microtubule-associated protein 1S & + & + & + & + & + & + \\
\hline $\mathrm{Ncl}$ & Nucleolin & +++ & +++ & +++ & +++ & +++ & +++ \\
\hline Nek8 & Serine/threonine-protein kinase Nek8 & $\cdot$ & $\cdot$ & $\cdot$ & . & . & . \\
\hline Nme7 & Nucleoside diphosphate kinase 7 & ++ & ++ & ++ & ++ & ++ & ++ \\
\hline Nphp3 & Nephronophthisis 3 & . & . & . & . & . & . \\
\hline Nrd1 & Nardilysin & +++ & +++ & +++ & +++ & +++ & +++ \\
\hline Nudcd1 & NudC domain-containing protein 1 & ++ & ++ & ++ & ++ & ++ & ++ \\
\hline Ogdh & Oxoglutarate dehydrogenase & ++ & ++ & ++ & ++ & ++ & ++ \\
\hline Osbpl3 & Oxysterol binding protein-like 3 & . & . & . & . & . & . \\
\hline $\mathrm{Pa2g} 4$ & Proliferation-associated protein $2 \mathrm{G} 4$ & +++ & +++ & +++ & +++ & +++ & +++ \\
\hline Pak2 & Serine/threonine-protein kinase PAK 2 & ++ & ++ & ++ & ++ & ++ & ++ \\
\hline Pdia3 & Protein disulfide-isomerase $\mathrm{A} 3$ & +++ & +++ & +++ & +++ & +++ & +++ \\
\hline Pin1 & Peptidyl-prolyl cis-trans isomerase NIMA-interacting 1 & . & . & . & . & . & . \\
\hline Рpp2ca & Protein phosphatase 2 , catalytic subunit alpha & +++ & +++ & +++ & +++ & +++ & +++ \\
\hline Prmt5 & Protein arginine $\mathrm{N}$-methyltransferase 5 & ++ & ++ & ++ & ++ & ++ & ++ \\
\hline Prom1 & Prominin 1 & . & . & . & . & . & . \\
\hline Psmb5 & Proteasome subunit beta type-5 & +++ & +++ & +++ & +++ & +++ & +++ \\
\hline Psmc1 & $26 S$ protease regulatory subunit 4 & +++ & +++ & +++ & +++ & +++ & +++ \\
\hline Psmd5 & 26S proteasome non-ATPase regulatory subunit 5 & ++ & ++ & ++ & ++ & ++ & ++ \\
\hline Ptges3 & Prostaglandin E synthase 3 & +++ & +++ & +++ & +++ & +++ & +++ \\
\hline Rab10 & Ras-related protein Rab10 & +++ & +++ & +++ & +++ & +++ & +++ \\
\hline Rab23 & Ras-related protein Rab23 & ++ & ++ & ++ & ++ & ++ & ++ \\
\hline Rab8a & Ras-related protein Rab-8A & ++ & ++ & ++ & ++ & ++ & ++ \\
\hline Rabl5 & Rab-like protein 5 & + & + & + & + & + & + \\
\hline Ran & RAN, member RAS oncogene family & +++ & +++ & +++ & +++ & +++ & +++ \\
\hline Ranbp1 & Ran-specific GTPase-activating protein & +++ & +++ & +++ & +++ & +++ & +++ \\
\hline Rpl4 & $60 S$ ribosomal protein $\mathrm{L} 4$ & +++ & +++ & +++ & +++ & +++ & +++ \\
\hline Rps14 & $40 \mathrm{~S}$ ribosomal protein $\mathrm{S} 14$ & +++ & +++ & +++ & +++ & +++ & +++ \\
\hline Rpsa & $40 S$ ribosomal protein SA & + & + & + & + & + & + \\
\hline Ruvbl2 & RuvB-like 2 & ++ & ++ & ++ & ++ & ++ & ++ \\
\hline Sars & Seryl-aminoacyl-tRNA synthetase & ++ & ++ & ++ & ++ & ++ & ++ \\
\hline $\operatorname{Sec} 24 b$ & Sec24 related gene family, member B & ++ & ++ & ++ & ++ & ++ & ++ \\
\hline Sept2 & Septin 2 & +++ & +++ & +++ & +++ & +++ & +++ \\
\hline Sept7 & Septin 7 & +++ & +++ & +++ & +++ & +++ & +++ \\
\hline Serbp1 & Serpine1 mRNA binding protein 1 & +++ & +++ & +++ & +++ & +++ & +++ \\
\hline Stk10 & Serine/threonine-protein kinase 10 & . & . & . & . & . & . \\
\hline Surf4 & Surfeit locus protein 4 & ++ & ++ & ++ & ++ & ++ & ++ \\
\hline Syncrip & Synaptotagmin binding RNA interacting protein & +++ & +++ & +++ & +++ & +++ & +++ \\
\hline Tpd52 & Tumor protein D52 & . & . & . & . & . & . \\
\hline Traf3ip1 & TRAF3-interacting protein 1 & + & + & + & + & + & + \\
\hline Ttc21b & Tetratricopeptide repeat domain 21B & + & + & + & + & + & + \\
\hline Ttc26 & Tetratricopeptide repeat protein 26 & . & . & . & . & . & . \\
\hline Tubb2b & Tubulin beta-2B chain & + & + & + & + & + & + \\
\hline Tubb5 & Tubulin beta- 5 chain & +++ & +++ & +++ & +++ & +++ & +++ \\
\hline Ube $4 b$ & Ubiquitin conjugation factor E4 B & ++ & ++ & ++ & ++ & ++ & ++ \\
\hline Usp14 & Ubiquitin carboxyl-terminal hydrolase 14 & +++ & +++ & +++ & +++ & +++ & +++ \\
\hline Wdr11 & WD repeat domain 11 & ++ & ++ & ++ & ++ & ++ & ++ \\
\hline Wdr19 & WD repeat domain 19 & + & + & + & + & + & + \\
\hline Wdr34 & WD repeat domain 34 & + & + & + & + & + & + \\
\hline Wdr35 & WD repeat domain 35 & + & + & + & + & + & + \\
\hline Wdr60 & WD repeat-containing protein 60 & + & + & + & + & + & + \\
\hline Xpo7 & Exportin 7 & ++ & ++ & ++ & ++ & ++ & ++ \\
\hline 1500003003 & Uncharacterized protein & + & + & + & + & + & + \\
\hline
\end{tabular}

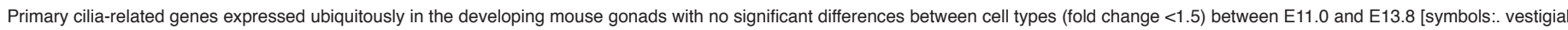
level of expression (1-6); + high level of expression (7-8); ++ strong expression (9-10); +++ very strong expression (11-12)]. 
indicates that indeed this primary cilia-related gene is involved in gonad development. The transcriptome analysis presented in this study creates a valuable database, which will be crucial in further studies of the role of primary cilia or their related genes in the development and/or differentiation of the gonads.

\section{Materials and Methods}

The study had been approved by the 1st Local Commission for Ethics in Experiments on Animals. Five transgenic mouse lines were used to isolate the supporting, interstitial/stromal and germ cells as previously

TABLE 2

PRIMARY CILIA-RELATED GENES EXPRESSED AT HIGHER LEVEL IN GERM CELLS COMPARED TO GONADAL SOMATIC CELLS

\begin{tabular}{|c|c|c|c|c|c|c|c|}
\hline \multirow[b]{2}{*}{ Gene symbol } & \multirow[b]{2}{*}{ Gene name } & \multicolumn{2}{|c|}{ Supporting cells } & \multicolumn{2}{|c|}{ Interstitial/stromal cells } & \multicolumn{2}{|c|}{ Germ cells } \\
\hline & & $\mathbf{x x}$ & $\mathbf{X Y}$ & $\mathbf{x X}$ & $\mathbf{X Y}$ & $\mathbf{x x}$ & $\mathbf{X Y}$ \\
\hline Asna1 & Arsenical pump-driving ATPase & + & + & + & + & ++ & ++ \\
\hline Diap1 & Protein diaphanous homolog 1 & + & + & + & + & ++ & ++ \\
\hline Ide & Insulin-degrading enzyme & + & + & + & + & +++ & +++ \\
\hline Ppp2r1b & Protein phosphatase 2 , regulatory subunit $A$, beta & ++ & ++ & ++ & ++ & +++ & +++ \\
\hline $\operatorname{Prd} 44$ & Peroxiredoxin 4 & ++ & ++ & ++ & ++ & +++ & +++ \\
\hline Rp/12 & $60 S$ ribosomal protein $\mathrm{L} 12$ & . & . & . & . & + & + \\
\hline Rplp2 & $60 S$ acidic ribosomal protein $\mathrm{P} 2$ & ++ & ++ & ++ & ++ & +++ & +++ \\
\hline Rps12 & Ribosomal protein $\mathrm{S} 12$ & + & + & + & + & +++ & +++ \\
\hline Slc2a1 & Solute carrier family 2 , member 1 & + & + & + & + & ++ & +++ \\
\hline Tsga14 & Testis specific gene A14 & . & . & . & . & ++ & ++ \\
\hline Tuba4a & Tubulin alpha-4A chain & . & . & . & . & ++ & ++ \\
\hline Xpo5 & Exportin 5 & ++ & ++ & ++ & ++ & +++ & +++ \\
\hline
\end{tabular}

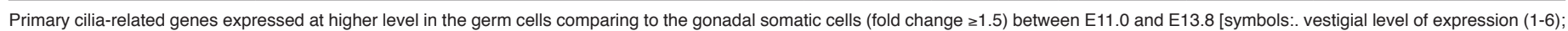
+ high level of expression (7-8); ++ strong expression (9-10); +++ very strong expression (11-12)].

TABLE 3

PRIMARY CILIA-RELATED GENES EXPRESSED AT LOWER LEVEL IN GERM CELLS COMPARED TO GONADAL SOMATIC CELLS

\begin{tabular}{|c|c|c|c|c|c|c|c|}
\hline \multirow[b]{2}{*}{ Gene symbol } & \multirow[b]{2}{*}{ Gene name } & \multicolumn{2}{|c|}{ Supporting cells } & \multicolumn{2}{|c|}{ Interstitial/stromal cells } & \multicolumn{2}{|c|}{ Germ cells } \\
\hline & & $\mathbf{X X}$ & $\mathrm{XY}$ & $\mathbf{X X}$ & $\mathbf{X Y}$ & $\mathbf{x X}$ & $\mathbf{X Y}$ \\
\hline Arl3 & ADP-ribosylation factor-like protein 3 & ++ & ++ & ++ & ++ & + & + \\
\hline Camk2d & Calcium/calmodulin-dependent protein kinase II, delta & ++ & ++ & ++ & ++ & . & . \\
\hline Capn2 & Calpain-2 catalytic subunit & ++ & ++ & ++ & ++ & . & . \\
\hline Erbb2ip & Erbb2 interacting protein & +++ & +++ & +++ & +++ & ++ & ++ \\
\hline Gnas & GNAS (guanine nucleotide binding protein) & ++ & ++ & ++ & ++ & + & + \\
\hline ldh1 & Isocitrate dehydrogenase 1 & +++ & +++ & +++ & +++ & ++ & ++ \\
\hline $\operatorname{Prd} \times 3$ & Peroxiredoxin 3 & ++ & ++ & ++ & ++ & + & + \\
\hline Ptpn13 & Protein tyrosine phosphatase, non-receptor type 13 & ++ & ++ & ++ & ++ & + & + \\
\hline Sec23a & Protein transport protein Sec23A & ++ & ++ & ++ & ++ & + & + \\
\hline Sept9 & Septin 9 & ++ & ++ & ++ & ++ & + & + \\
\hline Tpi1 & Triosephosphate isomerase & ++ & ++ & ++ & ++ & + & + \\
\hline Ttc30b & Tetratricopeptide repeat protein $30 \mathrm{~B}$ & ++ & ++ & ++ & ++ & + & + \\
\hline Tubb2a & Tubulin beta- $2 A$ chain & +++ & +++ & +++ & +++ & + & + \\
\hline
\end{tabular}

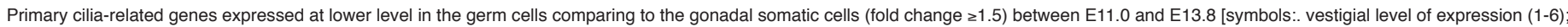

+ high level of expression (7-8); ++ strong expression (9-10); +++ very strong expression (11-12)].

TABLE 4

PRIMARY CILIA-RELATED GENES SHOWING SIGNIFICANT DIFFERENCES IN EXPRESSION LEVELS BETWEEN DIFFERENT CELL TYPE OR SEX

\begin{tabular}{|c|c|c|c|c|c|c|c|}
\hline \multirow[b]{2}{*}{ Gene symbol } & \multirow[b]{2}{*}{ Gene name } & \multicolumn{2}{|c|}{ Supporting cells } & \multicolumn{2}{|c|}{ Interstitial/stromal cells } & \multicolumn{2}{|c|}{ Germ cells } \\
\hline & & $x \mathbf{x}$ & $\mathbf{X Y}$ & $\mathbf{x x}$ & $\mathbf{X Y}$ & $\mathbf{x x}$ & $\mathrm{XY}$ \\
\hline Rangap1 & Ran GTPase-activating protein 1 & + & ++ & + & + & + & + \\
\hline Sec23b & Protein transport protein Sec23B & + & ++ & + & + & + & + \\
\hline Sntb2 & Syntrophin, basic 2 & + & ++ & + & + & + & + \\
\hline Tmem2 & Transmembrane protein 2 & + & ++ & + & + & + & + \\
\hline Tubb3 & Tubulin beta- 3 chain & . & ++ & . & . & . & . \\
\hline Tubb6 & Tubulin beta- 6 chain & . & . & ++ & ++ & . & . \\
\hline
\end{tabular}

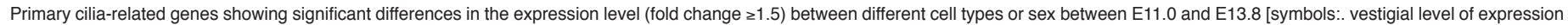
(1-6); + high level of expression (7-8); ++ strong expression (9-10); +++ very strong expression (11-12)]. 
described (Piprek et al., 2017). All individuals were genotyped to define sex and the presence of transgene as previously described (Piprek et al., 2017). The gonads from mouse fetuses were pooled accordingly to the sex and developmental stage. The gonads were incubated in $250 \mu \mathrm{l} 0.25 \%$ Trypsin-EDTA (Sigma, \#T4049) at $37^{\circ} \mathrm{C}$ for 5-10 minutes (Piprek et al., 2017). After tissue dissociation, the enzyme solution was replaced with PBS. Cells were centrifuged and the cell pellet was resuspended in PBS with Hoechst dye and incubated for $15 \mathrm{~min}$. About 100,000 cells were isolated from one sample. Pooled gonads from 5 fetuses were used for each time point and experiments were repeated three times. Fluorescence-activated cell sorting (FACS) was used to segregate three cell types isolated from the gonads (Piprek et al., 2017). Total RNA was isolated from each cell type and analyzed using microarray technique as previously described (Piprek et al., 2017). Raw data were analyzed as previously described (Piprek et al., 2017), and normalized data are available in Gene Expression Omnibus (accession number GSE94806). Data obtained by microarray analysis were confirmed by real-time quantitative PCR (qPCR) of eight chosen genes. 50 ng RNA of each sample was reverse-transcribed into cDNA using random primers and SuperScript III Reverse Transcriptase (Invitrogen, 18080044). The qPCR procedure was performed in $5 \mu$ reactions using SYBR Green Master Mix (Life Technologies, 4312704) in the 7500 Fast Real-Time PCR System (Applied Biosystems) with universal cycling parameters and analyzed as previously described (Svingen et al., 2009; Piprek et al., 2017). Statistical analysis was performed using the nonparametric ANOVA Kruskal-Wallis test, Tukey's test and Statistica 7.0 software.

\section{Acknowledgements}

The study was conducted within the project financed by the National Science Centre assigned on the basis of the decision \# DEC-2013/11/D/ NZ3/00184.

\section{References}

HIGGINBOTHAM H, EOM TY, MARIANI LE, BACHLEDA A, HIRT J, GUKASSYAN V, CUSACK CL, LAI C, CASPARY T, ANTON ES (2012). Arl13b in primary cilia regulates the migration and placement of interneurons in the developing cerebral cortex. Dev Cell 23: 925-938.
HU YC, OKUMURA LM, PAGE DC (2013). Gata4 is required for formation of the genital ridge in mice. PLoS Genet 9: e1003629.

ISHIKAWA H, THOMPSON J, YATES JR 3RD, MARSHALL WF (2012). Proteomic analysis of mammalian primary cilia. Curr Biol 22: 414-419.

NEL-THEMAAT L, VADAKKAN TJ, WANG Y, DICKINSON ME, AKIYAMA H, BEHRINGER RR (2009). Morphometric analysis of testis cord formation in Sox9-EGFP mice. Dev Dyn 238: 1100-1110.

NYGAARD MB, ALMSTRUP K, LINDBAEKL, CHRISTENSEN ST, SVINGENT(2015). Cell context-specific expression of primary cilia in the human testis and ciliary coordination of Hedgehog signalling in mouse Leydig cells. Sci Rep 5: 10364.

OU Y, DORES C, RODRIGUEZ SOSA JR, VAN DER HOORN F, DOBRINSKI I (2014). Primary cilia in the developing pig testis. Cell Tissue Res 358: 597-605.

PIPREK RP (2009a). Genetic mechanisms underlying male sex determination in mammals. J Appl Genet 50: 347-360.

PIPREK RP (2009b). Molecular mechanisms underlying female sex determination-antagonism between female and male pathway. Folia Biol 57: 105-113.

PIPREK RP (2010). Molecular machinery of gonadal differentiation in mammals. Int J Dev Biol 54: 779-786.

PIPREK RP, KLOC M, KUBIAK JZ (2016). Early development of the gonads: origin and differentiation of the somatic cells of the genital ridges. Results Probl Cell Differ 58: 1-22.

PIPREK RP, KOLASA M, PODKOWA D, KLOC M, KUBIAK JZ (2017). Cell adhesion molecules expression pattern indicates that somatic cells arbitrate gonadal sex of differentiating bipotential fetal mouse gonad. Mech Dev 147: 17-27.

PIPREK RP, KOLASAM, PODKOWAD, KLOC M, KUBIAK JZ (2018). Transcriptional profiling validates involvement of extracellular matrix and proteinases genes in mouse gonad development. Mech Dev 149: 9-19.

SATIR P, PEDERSEN LB, CHRISTENSEN ST (2010). The primary cilium at a glance. J Cell Sci 123: 499-503.

WAINWRIGHT EN, SVINGEN T, NG ET, WICKING C, KOOPMAN P (2014). Primary cilia function regulates the length of the embryonic trunk axis and urogenital field in mice. Dev Biol 395: 342-354. 


\section{Further Related Reading, published previously in the Int. J. Dev. Biol.}

Left-right asymmetry specification in amphioxus: review and prospects

Vladimir Soukup

Int. J. Dev. Biol. (2017) 61: 611-620

https://doi.org/10.1387/ijdb.170251vs

Roles of the cilium-associated gene CCDC11 in left-right patterning and in laterality disorders in humans Michal Gur, Enbal Ben-Tal Cohen, Olga Genin, Abraham Fainsod, Zeev Perles and Yuval Cinnamon

Int. J. Dev. Biol. (2017) 61: 267-276

https://doi.org/10.1387/ijdb.160442yc

All in one - integrating cell polarity, meiosis, mitosis and mechanical forces in early oocyte differentiation in vertebrates

Yaniv M. Elkouby

Int. J. Dev. Biol. (2017) 61: 179-193

https://doi.org/10.1387/ijdb.170030ye

Prevalence and genetics of immotile-cilia syndrome and left-handedness

Bjorn A. Afzelius and Unne Stenram

Int. J. Dev. Biol. (2006) 50: 571-573

https://doi.org/10.1387/ijdb.052132ba

\section{Asymmetry of cilia and of mice and men}

B A Afzelius

Int. J. Dev. Biol. (1999) 43: 283-286

http://www.intjdevbiol.com/web/paper/10470644

Situs inversus and ciliary abnormalities. What is the connection?

B A Afzelius

Int. J. Dev. Biol. (1995) 39: 839-844

http://www.intjdevbiol.com/web/paper/8645568

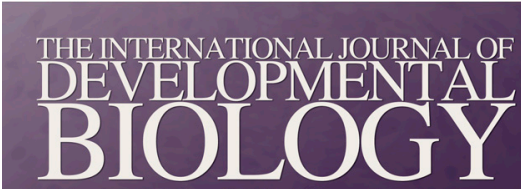

Volume 60 Nos. 10/11/12 Special Issue

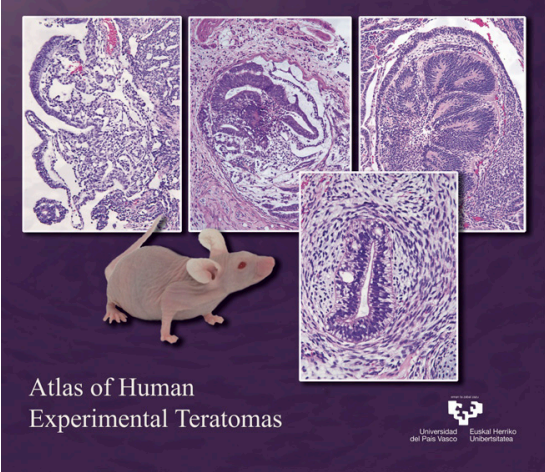

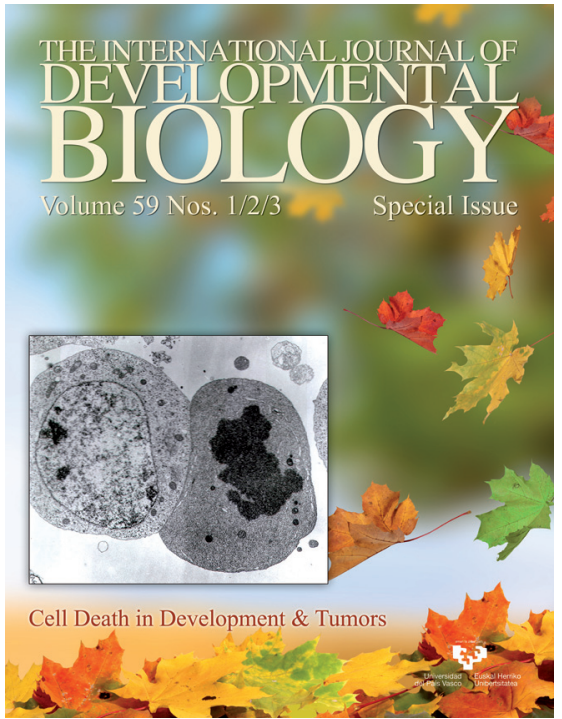
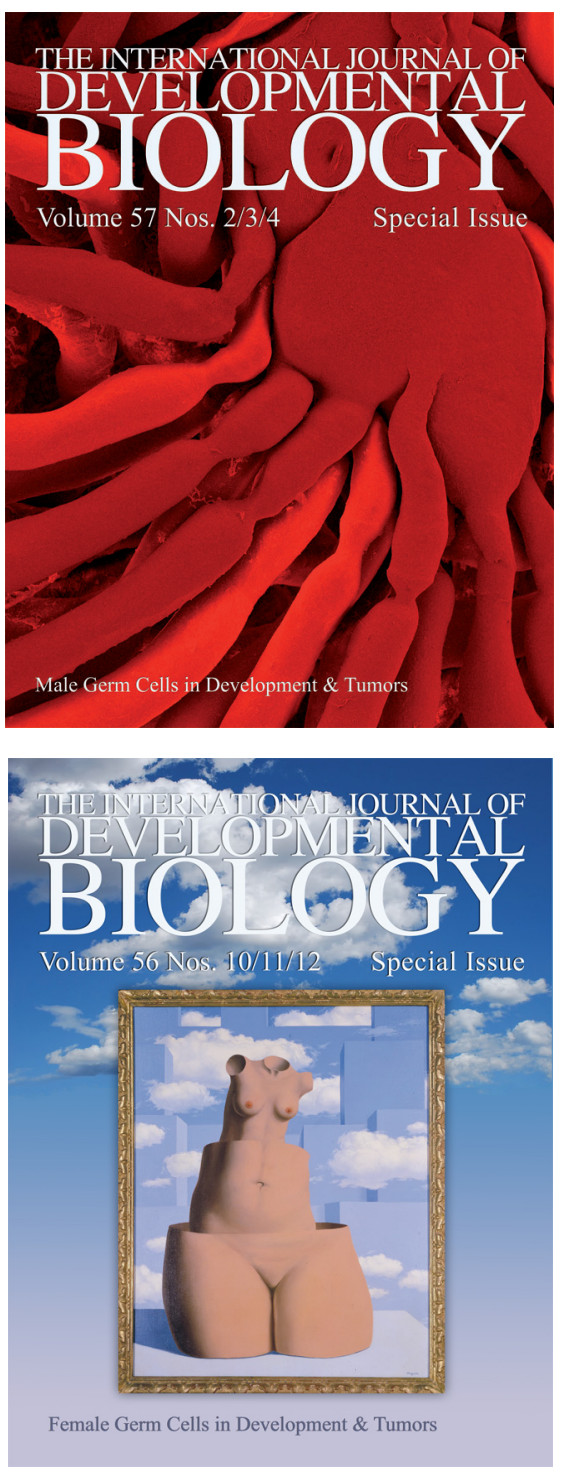